\section{Unsteady Motion of Continuous Media}

By Prof. K. P. Stanyukovich. Translation edited by Prof. Maurice Holt. Literal translation by J. George Adashko. Pp. xiii + 745. (London and New York: Pergamon Press, 1960.) 100s. net.

$\mathrm{TXCEPT}$ for two chapters which deal with conC densed media, this is a book on gas dynamies, covering approximately the same ground as Courant and Friedrich's Supersonic Flow and Shock Waves, with tho addition of some material of interest in astrophysical problems. The basic mathematics and thermodynamies of gas dynamies, and the theories of plane, cylindrical and spherical shock and detonation waves are all very thoroughly treated; in some respects too thoroughly, for the description is very detailed and at times repetitive. The volume might well have been condensed a great deal without sacrificing any essential material.

It was felt that the interest and value of the book would have been increased if more reference had been made to the many physical and technical problems to which the theory can be applied. The author confines himself almost entirely to problems which are amenable to exact mathematical solution; numerical methods, which are so frequently required in applications of this theory, are scarcely mentioned. Nevertheless, a great deal of material, worked out in detail, is included, and the editor and translator have performed a valuable service in making this available to English-speaking research workers in gas dynamics. Unfortunately, the value of the book as a guide to Russian literature in this field is diminished by the inadequacy of most of the references to original work.

A. J. M. Spencer

\section{Basic Essentials of Blood Group Theory and} Practice

By Kathleen E. Boorman and Barbara E. Dodd. Pp. viii $+133+8$ plates. (London: $J$. and $A$. Churchill, 1961.) 18s. net.

A $\mathrm{S}$ explained in the introduction, this book consists of extracts from the forthcoming second edition of the authors' An Introduction to Blood Group Serology. The present work is said to be intended for the non-specialist serologist or technician who is responsible for the routine serology in a hospital laboratory, and for students taking the intermediate examination in blood group serology of the Institute of Medical Laboratory Technology.

The book contains instructions on equipment and reagents, and on methods of $\mathrm{ABO}$ and $\mathrm{Rh}$ testing and direct matehing of blood. The authors are two of the leading British blood-group workers, and their practical experience is shown in their choice of the tests to be included and in tho elarity of the practical instructions. It is, however, surprising to find in a book published in 1961 that the first and second methods described for ABO grouping are respectively the open-tile method and the opon-slide method, procedures which, except as an occasional rapid preliminary to a definitive tube test, ought to be regarded as obsolete. Here, unfortunately, it was probably felt that they must be included for examination purposes.

In a book for members of medical auxiliary professions, whether nurses or technicians, it is always difficult to docide how much elinical information should be included. The inclusion, in this book, of clinical details of hæmolytic disease of the new-born undoubtedly adds to its interest, but it must not be allowed to tempt technicians into proffering clinical advice to patients or junior doctors.

The illustrations are exceptionally good; there are a glossary and an adequate index. The thin covers may, however, soon become torn on the laboratory bench. Nevertheless this book can be recommended warmly to the workers for whom it is written.

\section{A. E. Mourant}

\section{Astronomy}

By Patrick Moore. Pp. 253. (London: Oldbourne Book Co., Ltd., 1961.) 35s.

7 HIS is a picture book of astronomy. It has strong eye-appeal, the most engaging features being the colour photographs of star fields and gaseous nebulæe recently taken at Mount Palomar. Black-and-white photographs of heavenly objects, which have been the cherished possessions of astronomy students, are well represented too. The reproduction is excellent. There are many drawings of the planets, which are very attractive. Further, there is a good gallery of old drawings, ancient instruments, places and portraits of astronomical interest. The text is adequate everywhere, and is supported by many well-drawn and informative diagrams. The author states in the foreword that "this is a book for amateurs"; it is certainly one of the best of its kind.

T. KIANG

\section{Encyclopaedic Dictionary of Physics}

General, Nuclear, Solid State, Molecular, Chemical, Metal and Vacuum Physics, Astronomy, Geophysics, Biophysics and Related Subjects. Edited by J. Thewlis, in association with R. C. Glass, D. J. Hughes and A. R. Meetham. Vol. 1: A to Compensated Bars. Pp. $x v+800$. (London and New York: Pergamon Press, 1961.) $£ 100$ or 300 dollars per set.

A DICTIONARY of physics, if it is to be useful $A$ at all, must be as near perfection as possible. In attempting such perfection, the compilers of this work have sought the aid of a large number of authorities, each of whom-with a few exceptions-has signed the article he has written, even if it consists of only a few words. The result, as judged by the first volume, seems to be eminently successful.

It is much more than a dictionary. There are many entries of two pages or so: some of them are written for the expert; others, such as that on "Age Determination by Radioactivity", make interesting and informative reading. The term 'encyclopædic' is therefore well deserved.

Without a long period of use it is difficult to assess the real utility of the work. From the first volume it would appear that there is too little cross-indexing. For example, to find the item just mentioned, most people would first of all have looked up 'radioactive dating', not 'age determination'. It is to be hoped that difficulties of this sort will be catered for by a good index.

The proof-reading seems to have been excellently done, and there are few obvious errors. It is a pity, however, that the article on absolute zero does not give the value on the centigrade scale. Ampere does not have his correct accent, and the first entry of all gives Abbe an accent which denies him his German origin.

The work is beautifully printed and produced, and it can be thoroughly recommended.

H. LIPSON 